\title{
Association of microRNA Polymorphisms with the Risk of Myocardial Infarction in a Chinese Population
}

\author{
Cunrong Chen, ${ }^{1, *}$ Huashan Hong, ${ }^{2, *}$ Lianglong Chen, ${ }^{3}$ Xiaoyun Shi, ${ }^{2}$ Ying Chen ${ }^{2}$ \\ and Qinyong Weng ${ }^{1}$
}

\author{
${ }^{1}$ Department of Critical Care Medicine, Union Hospital, Fujian Medical University, Fuzhou, Fujian, P.R. China \\ ${ }^{2}$ Senior Officials Inpatient Ward, Union Hospital, Fujian Medical University, Fuzhou, Fujian, P.R. China \\ ${ }^{3}$ Department of Cardiology, Union Hospital, Fujian Medical University, Fuzhou, Fujian, P.R. China
}

\begin{abstract}
MicroRNAs (miRNAs) are involved in the regulation of a variety of biological processes, such as inflammation. Dysregulation of miRNAs have been implicated in many human disease, including cardiovascular diseases. Polymorphisms in miRNA genes may affect the miRNA biogenesis and function, and thus cause changes in the expression of thousands of genes. The aim of this study was to examine whether miRNA polymorphisms (miR-146a rs2910164, miR-149 rs71428439, miR-196a2 rs11614913, miR-218 rs11134527, and miR-499 rs3746444) contribute to the risk for the development of myocardial infarction (MI). Five miRNA polymorphisms were genotyped in a total of 1808 subjects composed of 919 MI patients and 889 control individuals. The GG genotype of rs3746444 was found to be associated with a significantly increased risk of $\mathrm{MI}$ (recessive model, adjusted $\mathrm{OR}=1.710,95 \% \mathrm{Cl}: 1.058-2.763, P=0.029$ ). Although the CC genotype of rs2910164 significantly increased the risk of MI under dominant and additive models $(P<0.05)$, this difference disappeared after adjustment for age, sex, blood pressure, triglycerides, total cholesterol, HDL, LDL and diabetes. In addition, when rs3746444 and rs2910164 were evaluated together by the number of putative high-risk alleles, we found an increased risk of $\mathrm{Ml}$ for subjects carrying 3-4 risk alleles (3-4 risk alleles vs. 0-1 risk allele, adjusted OR $=1.580,95 \% \mathrm{Cl}: 1.069-2336, P=0.022 ; 3-4$ risk alleles vs. $0-2$ risk allele, adjusted $\mathrm{OR}=1.513,95 \% \mathrm{Cl}: 1.031-2.219, P=0.034)$. These findings indicate that miR-499 rs3746444 and miR-146a rs2910164 may represent novel markers of MI susceptibility.
\end{abstract}

Keywords: microRNA; miR-146a; miR-499; myocardial infarction; susceptibility Tohoku J. Exp. Med., 2014 June, 233 (2), 89-94. (C) 2014 Tohoku University Medical Press

\section{Introduction}

Myocardial infarction (MI) is a leading cause of death and disability worldwide, approximately 2.9 million Chinese suffering MI each year (Gu et al. 2014). Despite considerable advances in diagnosis and treatment strategies, consequences of MI for families and society are enormous and costly due to its high mortality and disability rates. In-hospital and 30-day mortality after an acute MI has declined over the past decades with the invention of coronary care units, reperfusion therapy and evidence based medicines (Ford et al. 2007). Many extrinsic and intrinsic factors for MI have been identified, such as smoking, hypertension, diabetes and hyperlipidaemia. To date, the exact pathophysiological mechanisms leading to the development of MI remain poorly understood, but it is generally believed that the complex interaction of susceptibility genes with other genetic and environmental risk factors contribute to the occurrence of MI (Guella et al. 2009).

MicroRNAs (miRNAs) are a family of approximate 18-25 nt, highly conserved, non-coding, single stranded RNAs that regulate gene expression at post-transcriptional level. miRNAs bind to the 3 '-untranslated region of their target mRNAs, resulting in mRNA cleavage and translational repression (Bartel 2009). There are more than 2,500 mature human miRNA sequences in the miRBase database (release 20.0) (Kozomara and Griffiths-Jones 2011). It has been suggested that each miRNA potentially regulates hundreds of target genes (Wu et al. 2014), and miRNAs are thus likely involved in almost all biological processes, such as cell proliferation, cell proliferation, differentiation, apoptosis and stress resistance (Yu et al. 2013; Wu et al. 2014). It is not surprising that abnormal levels of miRNAs have been implicated in many human disease, including cancer

Received March 24, 2014; revised and accepted April 30, 2014. Published online May 22, 2014; doi: 10.1620/tjem.233.89.

*These two authors contributed equally to this work.

Correspondence: Huashan Hong, M.D., Ph.D., Senior Officials Inpatient Ward, Union Hospital, Fujian Medical University, No. 29

Xinquan Road, Fuzhou, Fujian 350001, P.R. China.

e-mail: honghuashan@medmail.com 
(Yu et al. 2014) and cardiovascular diseases (Condorelli et al. 2014). Growing evidence has demonstrated that miRNAs participate in the regulation of glucose, cholesterol and lipid metabolism, proliferation of vascular smooth muscle cells and inflammation, which play important roles in the development and progression of cardiovascular diseases (Braunwald 2013; Vickers et al. 2014). Recent studies have been demonstrated that aberrant expression of miRNAs has been involved in pathogenesis of MI (Vickers et al. 2014; Sala et al. 2014).

Since single nucleotide polymorphisms (SNPs) located in pre-miR genes may directly affect the miRNA maturation and expression, and the binding to target mRNAs, and subsequently affect the expression of target genes (Wu et al. 2014). Therefore, a function SNP in miRNA genes may affect many signaling pathways by altering the expression levels of thousands of genes (Wu et al. 2014). Previous studies have shown that SNPs in miRNA genes are associated with the susceptibility to many disease, including cancer (Omrani et al. 2014). Previous studies revealed that miR-146a rs2910164 (Xiong et al. 2014), miR-149 rs71428439 (Ding et al. 2013), miR-196a2 rs11614913 (Zhou et al. 2010; Zhi et al. 2012), and miR-499 rs3746444 (Zhi et al. 2012; Liu et al. 2014) were related to the risk of cardiovascular diseases, including coronary artery disease (CAD), ischemic stroke and MI. Furthermore, miR-218 plays an important role in heart development (Fish et al. 2011; Chiavacci et al. 2012). Rs11134527 G allele in the miR-218 precursor causes an alteration in the step-loop structure and increases the expression of mature miR-218 (Gao et al. 2013). Therefore, our aim was to evaluate whether 5 miRNA SNPs (miR-146a rs2910164, miR-149 rs71428439, miR-196a2 rs11614913, miR-218 rs11134527, and miR-499 rs3746444) determine the susceptibility to MI.

\section{Materials and Methods}

\section{Subjects}

The study including 919 Chinese individuals with MI who were referred to Union Hospital. The diagnosis of definite MI was based on typical ECG changes, elevated cardiac markers, clinical history, and coronary angiography (stenosis $\geq 50 \%$ ). The control group included 889 unrelated individuals, who were judged to be free of any history of cardiac disease by questionnaires, medical history, and clinical examination. All subjects were unrelated Han Chinese. This study adhered to the principles of the Declaration of Helsinki, and all participants gave written informed consent to take part in the study, according to the process approved by the Ethical Committee of Union Hospital.

\section{Genotyping}

Genomic DNA was isolated from peripheral blood leukocytes using Flexigene DNA kit (Qiagen, Hilden, Germany) according to the manufacturer's instructions. Genotyping was performed by polymerase chain reaction-ligation detection reaction (PCR-LDR) method (Zhang et al. 2012). For quality control, 96 randomly selected samples were re-genotyped by both PCR-LDR and DNA sequencing.
The PCR-LDR gave results that were $100 \%$ concordant with DNA sequencing on all 5 SNPs.

\section{Statistical analyses}

Basic and clinical variables are mentioned as mean \pm S.D. for the quantitative variables, and are summarized using frequency (percentage) for the categorical variables. Comparison of these variable between patients and controls was performed by Student $t$ test, nonparametric Mann-Whitney test and chi-square test. The HardyWeinberg equilibrium was tested by a chi-square test. Odds ratios (ORs) and 95\% confidence intervals (CIs) were calculated by using univariate logistic regression. In the multivariate analysis, ORs and 95\% CIs were adjusted for age, sex, systolic blood pressure, diastolic blood pressure, triglycerides, total cholesterol, high-density lipoprotein cholesterol (HDL), low-density lipoprotein cholesterol (LDL) and diabetes. A $P$ value $<0.05$ was considered as a significant criterion. All statistical analyses were carried out by SPSS, version 20 (SPSS, Inc., Chicago, IL, USA).

\section{Results}

The clinical characteristics were summarized in Table 1, including age, sex, blood pressure, and blood lipid. We evaluated genotype frequencies of SNPs in miRNA genes in case and control subjects and their associations with the susceptibility of MI. The genotype distribution of 5 SNPs in control group did not differ significantly from HardyWeinberg equilibrium $(P>0.05)$. The genotype frequencies of rs 3746444 were $7.6 \%(\mathrm{GG}), 25.8 \%$ (AG) and $66.6 \%$ (AA) among cases, and $4.2 \%$ (GG), 27.7\% (AG) and 68.2\% (AA) among controls, and the difference was statistically significant $(P=0.007)$. Logistic regression analyses showed that the GG genotype of rs3746444 was significantly associated with an increased risk of MI compared with the AA genotype (OR $=1.873,95 \%$ CI: $1.238-2.834$, $P=0.003$ ) (Table 2). The difference remained significant even after adjustment for age, sex, systolic blood pressure, diastolic blood pressure, triglycerides, total cholesterol, HDL, LDL and diabetes (OR $=1.745,95 \%$ CI: 1.074-2.836, $P=0.025)$. In addition, among three genetic models assayed, the GG genotype of rs3746444 showed a significant association with the disease only under recessive model (adjusted OR $=1.710,95 \%$ CI: 1.058-2.763, $P=$ 0.029). Although rs 2910164 was significantly associated with an increased risk of MI (CC vs. GG, OR $=1.368,95 \%$ CI: 1.044-1.791, $P=0.023$; dominant model, $\mathrm{OR}=1.237$, 95\% CI: $1.014-1.509, P=0.036$; additive genetic model, $\mathrm{OR}=1.172,95 \% \mathrm{CI}: 1.027-1.338, P=0.019$ ), we found no significant association of rs2910164 with MI risk after adjustment for age, sex, systolic blood pressure, diastolic blood pressure, triglycerides, total cholesterol, HDL, LDL and diabetes $(P>0.05)$. No statistically significant difference was found between other 3 SNPs and MI $(P>0.05)$.

We further examined the combined effect of the cumulative number of putative risk alleles (rs3746444 $\mathrm{G}$ and rs2910164 C) on MI risk. A significant trend was observed between an increased number of risk alleles and an increased risk of $\mathrm{MI}(P=0.003$ for trend). Individuals with 
Table 1. Clinical characteristic of cases and controls.

\begin{tabular}{lccc}
\hline \multicolumn{1}{c}{ Clinical characteristics } & Cases $(\%)$ & Controls $(\%)$ & $P$ value \\
\hline Total & 919 & 889 & \\
Age (years) & $60.7 \pm 10.2$ & $59.9 \pm 10.3$ & 0.085 \\
Sex, \%male & 60.3 & 58.2 & 0.363 \\
Systolic blood pressure, $\mathrm{mmHg}$ & $139.8 \pm 17.7$ & $127.9 \pm 18.2$ & $<0.001$ \\
Diastolic blood pressure, $\mathrm{mmHg}$ & $82.6 \pm 11.8$ & $79.4 \pm 10.4$ & $<0.001$ \\
Triglycerides, mmol/L & $2.0 \pm 1.4$ & $1.8 \pm 1.1$ & 0.004 \\
Total cholesterol, mmol/L & $4.8 \pm 1.2$ & $4.7 \pm 1.0$ & 0.400 \\
HDL, mmol/L & $1.2 \pm 0.4$ & $1.2 \pm 0.3$ & $<0.001$ \\
LDL, mmol/L & $2.8 \pm 1.1$ & $2.5 \pm 0.6$ & $<0.001$ \\
ApoA & $1.3 \pm 0.2$ & $1.3 \pm 0.2$ & $<0.001$ \\
ApoB & $0.9 \pm 0.3$ & $0.9 \pm 0.2$ & $<0.001$ \\
Lp(a) & $0.3 \pm 0.5$ & $0.2 \pm 0.3$ & $<0.001$ \\
Diabetes*, $\%$ & 17.0 & 3.8 & $<0.001$ \\
\hline
\end{tabular}

*fasting blood glucose $\geq 7.0 \mathrm{mmol} / \mathrm{L}$.

Table 2. Associations between 5 SNPs and MI risk.

\begin{tabular}{|c|c|c|c|c|c|c|c|c|}
\hline miRNA & SNP & Genotype & Case & Control & Crude OR $(95 \% \mathrm{CI})$ & $P$ value & Adjusted OR $(95 \% \mathrm{CI}) *$ & $P$ value* \\
\hline \multirow[t]{6}{*}{ miR-149 } & rs71428439 & AA & $375(40.8)$ & $384(43.2)$ & 1 & & 1 & \\
\hline & & AG & $389(42.3)$ & 381 (42.9) & $1.046(0.856-1.278)$ & 0.664 & $0.905(0.651-1.258)$ & 0.552 \\
\hline & & GG & $155(16.9)$ & $124(13.9)$ & $1.280(0.972-1.686)$ & 0.079 & $1.126(0.886-1.431)$ & 0.333 \\
\hline & & \multicolumn{2}{|c|}{ Dominant model } & & $1.103(0.915-1.330)$ & 0.303 & $0.940(0.752-1.177)$ & 0.591 \\
\hline & & \multicolumn{2}{|c|}{ Recessive model } & & $1.252(0.968-1.618)$ & 0.086 & $1.175(0.866-1.594)$ & 0.302 \\
\hline & & \multicolumn{2}{|c|}{ Additive model } & & $1.111(0.976-1.266)$ & 0.112 & $1.012(0.866-1.183)$ & 0.877 \\
\hline \multirow[t]{6}{*}{$\operatorname{miR}-146 a$} & rs2910164 & GG & $269(29.3)$ & $301(33.9)$ & 1 & & 1 & \\
\hline & & $\mathrm{CG}$ & $463(50.4)$ & 435 (48.9) & $1.148(0.894-1.475)$ & 0.279 & $1.215(0.944-1.565)$ & 0.130 \\
\hline & & $\mathrm{CC}$ & $187(20.3)$ & $153(17.2)$ & $1.368(1.044-1.791)$ & 0.023 & $1.343(0.968-1.864)$ & 0.078 \\
\hline & & \multicolumn{2}{|c|}{ Dominant model } & & $1.237(1.014-1.509)$ & 0.036 & $1.247(0.981-1.586)$ & 0.072 \\
\hline & & \multicolumn{2}{|c|}{ Recessive model } & & $1.229(0.970-1.557)$ & 0.088 & $1.188(0.892-1.581)$ & 0.239 \\
\hline & & \multicolumn{2}{|c|}{ Additive model } & & $1.172(1.027-1.338)$ & 0.019 & $1.166(0.993-1.370)$ & 0.061 \\
\hline \multirow[t]{6}{*}{$\operatorname{miR}-218$} & rs11134527 & AA & $327(35.6)$ & $306(34.4)$ & 1 & & 1 & \\
\hline & & AG & $418(45.5)$ & $436(49.0)$ & $0.897(0.730-1.102)$ & 0.301 & $1.215(0.880-1.677)$ & 0.237 \\
\hline & & GG & $174(18.8)$ & $147(16.5)$ & $1.108(0.846-1.450)$ & 0.457 & $1.010(0.790-1.291)$ & 0.934 \\
\hline & & \multicolumn{2}{|c|}{ Dominant model } & & $0.950(0.783-1.153)$ & 0.605 & $1.062(0.842-1.338)$ & 0.612 \\
\hline & & \multicolumn{2}{|c|}{ Recessive model } & & $1.179(0.926-1.502)$ & 0.182 & $1.208(0.904-1.613)$ & 0.202 \\
\hline & & \multicolumn{2}{|c|}{ Additive model } & & $1.025(0.900-1.168)$ & 0.709 & $1.086(0.929-1.271)$ & 0.300 \\
\hline \multirow[t]{6}{*}{ miR-196a2 } & rs11614913 & $\mathrm{TT}$ & $312(33.9)$ & $322(36.2)$ & 1 & & 1 & \\
\hline & & $\mathrm{CT}$ & $450(49.0)$ & $406(45.7)$ & $0.994(0.759-1.301)$ & 0.963 & $1.115(0.871-1.426)$ & 0.388 \\
\hline & & $\mathrm{CC}$ & $157(17.1)$ & $161(18.1)$ & $0.874(0.712-1.074)$ & 0.200 & $0.929(0.677-1.274)$ & 0.648 \\
\hline & & \multicolumn{2}{|c|}{ Dominant model } & & $1.051(0.954-1.158)$ & 0.312 & $1.058(0.840-1.333)$ & 0.632 \\
\hline & & \multicolumn{2}{|c|}{ Recessive model } & & $0.965(0.855-1.089)$ & 0.567 & $0.873(0.658-1.159)$ & 0.347 \\
\hline & & \multicolumn{2}{|c|}{ Additive model } & & $1.025(0.900-1.169)$ & 0.707 & $1.015(0.870-1.185)$ & 0.846 \\
\hline \multirow[t]{6}{*}{$\operatorname{mir}-499$} & rs3746444 & $\mathrm{AA}$ & $612(66.6)$ & $606(68.2)$ & 1 & & 1 & \\
\hline & & AG & $237(25.8)$ & $246(27.7)$ & $0.954(0.773-1.178)$ & 0.661 & $1.074(0.834-1.383)$ & 0.579 \\
\hline & & GG & $70(7.6)$ & $37(4.2)$ & $1.873(1.238-2.834)$ & 0.003 & $1.745(1.074-2.836)$ & 0.025 \\
\hline & & \multicolumn{2}{|c|}{ Dominant model } & & $1.074(0.882-1.308)$ & 0.476 & $1.170(0.924-1.482)$ & 0.191 \\
\hline & & \multicolumn{2}{|c|}{ Recessive model } & & $1.899(1.261-2.860)$ & 0.002 & $1.710(1.058-2.763)$ & 0.029 \\
\hline & & \multicolumn{2}{|c|}{ Additive model } & & $0.868(0.743-1.013)$ & 0.073 & $1.198(0.994-1.444)$ & 0.058 \\
\hline
\end{tabular}

*adjusted for age, sex, systolic blood pressure, diastolic blood pressure, triglycerides, total cholesterol, HDL, LDL and diabetes. 
Table 3. Combined effects of rs3746444 and rs2910164 on MI risk.

\begin{tabular}{ccccc}
\hline No. of risk allele & Case & Control & Adjusted OR (95\% CI)* & $P$ value* \\
\hline $0-1$ & $559(60.8)$ & $585(65.8)$ & 1 & 0.256 \\
2 & $258(28.1)$ & $238(26.8)$ & $1.158(0.899-1.490)$ & 0.022 \\
$3-4$ & $102(11.1)$ & $66(7.4)$ & $1.580(1.069-2336)$ & \\
$0-2$ & $817(88.9)$ & $823(92.6)$ & 1 & 0.034 \\
$3-4$ & $102(11.1)$ & $66(7.4)$ & $1.513(1.031-2.219)$ & \\
\hline
\end{tabular}

*adjusted for age, sex, systolic blood pressure, diastolic blood pressure, triglycerides, total cholesterol, HDL, LDL and diabetes.

3-4 risk alleles had 1.580 (95\% CI: 1.069-2336) times increased risk for MI compared with those with 0-1 risk allele (Table 3). When individuals with 0-2 risk alleles were combined into one group, the increased risk of MI for those with 3-4 risk alleles remained statistically significant (adjusted OR $=1.513,95 \%$ CI: $1.031-2.219, P=0.034$ ) after adjustment for age, sex, systolic blood pressure, diastolic blood pressure, triglycerides, total cholesterol, HDL, LDL and diabetes.

\section{Discussion}

In the present study, we investigated the relationship between 5 miRNA SNPs and MI in a Chinese population. We found that rs3746444 and rs2910164 may affect the susceptibility to MI. To the best of our knowledge, this is the first study to evaluate the correlation between miRNA SNPs and MI in a large population. Our findings implied that miRNA SNPs may play important roles in the development of MI.

The miR-499 gene is located in the intron 20 of human cardiac $\beta$-myosin heavy chain 7B gene (MYH7B), and is highly expressed in cardiac tissue under physiological condition (Shieh et al. 2011). It has been shown that miR-499 can protect cardiomyocyte from ischemia/reperfusioninduced apoptosis by inhibiting calcineurin-mediated dephosphorylation of dynamin-related protein-1, while the knock-down of miR-499 induces myocardial apoptosis and increases the infarct size (Wang et al. 2011). Exposure of cardiomyocytes to hypoxic and ischemic stress leads to down-regulation of miR-499 (Wang et al. 2011). In addition, miR-499 regulates the expression of inflammatory cytokines, including IL-17R $\beta$, IL-23 $\alpha$, IL-2R, IL-6, IL-2 and IL-18R (Hashemi et al. 2013). Rs3746444 is located in the stem region of the miR-499, and may damage the secondary structure stability and thus affect the miRNA maturation process and binding affinities to its target genes $(\mathrm{Hu}$ et al. 2009). It is reasonable that $\mathrm{rs} 3746444$ may contribute to the susceptibility to various diseases by regulating distinct sets of downstream genes. Many studies have shown that rs3746444 is correlated with risks of a variety of diseases, such as different types of cancer (Wang et al. 2013), rheumatoid arthritis (Hashemi et al. 2013), CAD (Zhi et al. 2012) and ischemic stroke (Liu et al. 2014). Previous stud- ies revealed that the GG and AG genotypes of rs3746444 conferred increased risk for CAD (Zhi et al. 2012) and ischemic stroke (Liu et al. 2014), respectively. However, Xiong et al. (2014) found no association between rs3746444 and CAD. In the present study, we found that $\mathrm{rs} 3746444 \mathrm{GG}$ genotype increased the risk of $\mathrm{MI}$ in a Chinese population, indicating that MI may share genetic risk factor with both CAD and ischemic stroke. Potential mechanism for such association might be due to impaired ability to inhibit apoptosis and inflammation among GG homozygotes compared with AA homozygotes.

miR-146a plays important roles in regulating the innate immunity and inflammatory responses (Rusca and Monticelli 2011; Ichii et al. 2012). Aberrant expression of miR-146a has been observed in a variety of disease, including cancer, autoimmune and inflammatory diseases, and infectious diseases (Li et al. 2010). Rs2910164 resides in the pre-miR-146 gene on human chromosome 5 and results in from a $\mathrm{G}: \mathrm{U}$ pair to a $\mathrm{C}: \mathrm{U}$ mismatch in the stem structure of the miR-146a precursor. The presence of this mismatch reduces the expression of mature miR-146a and thus affects target mRNA binding (Jazdzewski et al. 2008; Xu et al. 2008). Two previous studies have reported that miR-146a is expressed at higher levels in peripheral blood mononuclear cells of CAD patients with rs2910164 CC genotype (Xiong et al. 2014; Ramkaran et al. 2014). Furthermore, Xiong et al. (2014) found an association of rs2910164 CC genotype with an increased risk of CAD in Chinese, while Ramkaran et al. (2014) demonstrated inconsistence in the relationship between rs2910164 and CAD risk in young South African Indians. One potential explanation for this phenomenon may be genetic backgrounds among races. In this study, we also found an association of rs2910164 with MI, but this difference disappeared after adjustment for age, sex, systolic blood pressure, diastolic blood pressure, triglycerides, total cholesterol, HDL, LDL and diabetes. However, we observed a stronger cumulative effect of rs3746444 and rs2910164 on MI risk. Individuals who possess 3-4 risk alleles have an increased chance of developing MI. Therefore, when multiple causative variants and clinical factors are combined, the risk prediction for MI may be improved.

In conclusion, this study provides first evidence that 
miR-499 rs3746444 and miR-146a rs2910164 may be associated with MI susceptibility in Chinese Han population. Our study provides new genetic markers and new insights into MI. However, replication of our finding is needed to confirm this association by independent genetic studies with a larger sample size, and further functional analyses are also necessary to uncover the underlying mechanism.

\section{Acknowledgments}

We thank Yuanjirong for reading this paper and for their valuable suggestions.

\section{Conflict of Interest}

The authors declare no conflict of interest.

\section{References}

Bartel, D.P. (2009) MicroRNAs: target recognition and regulatory functions. Cell, 136, 215-233.

Braunwald, E. (2013) Cardiovascular science: opportunities for translating research into improved care. J. Clin. Invest., 123, 6-10.

Chiavacci, E., Dolfi, L., Verduci, L., Meghini, F., Gestri, G., Evangelista, A.M., Wilson, S.W., Cremisi, F. \& Pitto, L. (2012) MicroRNA 218 mediates the effects of Tbx5a overexpression on zebrafish heart development. PLoS One, 7, e50536.

Condorelli, G., Latronico, M.V. \& Cavarretta, E. (2014) microRNAs in cardiovascular diseases: current knowledge and the road ahead. J. Am. Coll. Cardiol., [Epub ahead of print].

Ding, S.L., Wang, J.X., Jiao, J.Q., Tu, X., Wang, Q., Liu, F., Li, Q., Gao, J., Zhou, Q.Y., Gu, D.F. \& Li, P.F. (2013) A premicroRNA-149 (miR-149) genetic variation affects miR-149 maturation and its ability to regulate the Puma protein in apoptosis. J. Biol. Chem., 288, 26865-26877.

Fish, J.E., Wythe, J.D., Xiao, T., Bruneau, B.G., Stainier, D.Y., Srivastava, D. \& Woo, S. (2011) A Slit/miR-218/Robo regulatory loop is required during heart tube formation in zebrafish. Development, 138, 1409-1419.

Ford, E.S., Ajani, U.A., Croft, J.B., Critchley, J.A., Labarthe, D.R., Kottke, T.E., Giles, W.H. \& Capewell, S. (2007) Explaining the decrease in U.S. deaths from coronary disease, 1980-2000. N. Engl. J. Med., 356, 2388-2398.

Gao, X., Yang, L., Ma, Y., Yang, J., Zhang, G., Huang, G., Huang, Q., Chen, L., Fu, F., Chen, Y., Su, D., Dong, Y., Ma, X., Lu, C. \& Peng, X. (2013) No association of functional variant in pri-miR-218 and risk of congenital heart disease in a Chinese population. Gene, 523, 173-177.

Gu, K., Chang, Y., Gao, B., Wan, F., Loisance, D. \& Zeng, Y. (2014) Development of ventricular assist devices in China: present status, opportunities and challenges. Eur. J. Cardiothorac. Surg., [Epub ahead of print].

Guella, I., Rimoldi, V., Asselta, R., Ardissino, D., Francolini, M., Martinelli, N., Girelli, D., Peyvandi, F., Tubaro, M., Merlini, P.A., Mannucci, P.M. \& Duga, S. (2009) Association and functional analyses of MEF2A as a susceptibility gene for premature myocardial infarction and coronary artery disease. Circ. Cardiovasc. Genet., 2, 165-172.

Hashemi, M., Eskandari-Nasab, E., Zakeri, Z., Atabaki, M., Bahari, G., Jahantigh, M., Taheri, M. \& Ghavami, S. (2013) Association of pre-miRNA-146a rs2910164 and pre-miRNA-499 rs3746444 polymorphisms and susceptibility to rheumatoid arthritis. Mol. Med. Rep., 7, 287-291.

Hu, Z., Liang, J., Wang, Z., Tian, T., Zhou, X., Chen, J., Miao, R., Wang, Y., Wang, X. \& Shen, H. (2009) Common genetic variants in pre-microRNAs were associated with increased risk of breast cancer in Chinese women. Hum. Mutat., 30, 79-84.

Ichii, O., Otsuka, S., Sasaki, N., Namiki, Y., Hashimoto, Y. \& Kon, Y. (2012) Altered expression of microRNA miR-146a correlates with the development of chronic renal inflammation. Kidney Int., 81, 280-292.

Jazdzewski, K., Murray, E.L., Franssila, K., Jarzab, B., Schoenberg, D.R. \& de la Chapelle, A. (2008) Common SNP in pre-miR146 a decreases mature miR expression and predisposes to papillary thyroid carcinoma. Proc. Natl. Acad. Sci. USA, 105, 7269-7274.

Kozomara, A. \& Griffiths-Jones, S. (2011) miRBase: integrating microRNA annotation and deep-sequencing data. Nucleic Acids Res., 39, D152-157.

Li, L., Chen, X.P. \& Li, Y.J. (2010) MicroRNA-146a and human disease. Scand. J. Immunol., 71, 227-231.

Liu, Y., Ma, Y., Zhang, B., Wang, S.X., Wang, X.M. \& Yu, J.M. (2014) Genetic polymorphisms in pre-microRNAs and risk of ischemic stroke in a Chinese population. J. Mol. Neurosci., 52, 473-480.

Omrani, M., Hashemi, M., Eskandari-Nasab, E., Hasani, S.S., Mashhadi, M.A., Arbabi, F. \& Taheri, M. (2014) hsa-mir-499 rs 3746444 gene polymorphism is associated with susceptibility to breast cancer in an Iranian population. Biomark. Med., 8, 259-267.

Ramkaran, P., Khan, S., Phulukdaree, A., Moodley, D. \& Chuturgoon, A.A. (2014) miR-146a polymorphism influences levels of miR-146a, IRAK-1, and TRAF-6 in young patients with coronary artery disease. Cell Biochem. Biophys., 68, 259-266.

Rusca, N. \& Monticelli, S. (2011) MiR-146a in Immunity and Disease. Mol. Biol. Int., 2011, 437301.

Sala, V., Bergerone, S., Gatti, S., Gallo, S., Ponzetto, A., Ponzetto, C. \& Crepaldi, T. (2014) MicroRNAs in myocardial ischemia: identifying new targets and tools for treating heart disease. New frontiers for miR-medicine. Cell. Mol. Life Sci., 71, 1439-1452.

Shieh, J.T., Huang, Y., Gilmore, J. \& Srivastava, D. (2011) Elevated miR-499 levels blunt the cardiac stress response. PLoS One, 6, e19481.

Vickers, K.C., Rye, K.A. \& Tabet, F. (2014) MicroRNAs in the onset and development of cardiovascular disease. Clin. Sci. (Lond), 126, 183-194.

Wang, J.X., Jiao, J.Q., Li, Q., Long, B., Wang, K., Liu, J.P., Li, Y.R. \& Li, P.F. (2011) miR-499 regulates mitochondrial dynamics by targeting calcineurin and dynamin-related protein-1. Nat. Med., 17, 71-78.

Wang, Z., Wu, J., Zhang, G., Cao, Y., Jiang, C. \& Ding, Y. (2013) Associations of miR-499 and miR-34b/c Polymorphisms with Susceptibility to Hepatocellular Carcinoma: An EvidenceBased Evaluation. Gastroenterol. Res. Pract., 2013, 719202.

Wu, C., Li, M., Hu, C. \& Duan, H. (2014) Prognostic role of microRNA polymorphisms in patients with advanced esophageal squamous cell carcinoma receiving platinum-based chemotherapy. Cancer Chemother. Pharmacol., 73, 335-341.

Xiong, X.D., Cho, M., Cai, X.P., Cheng, J., Jing, X., Cen, J.M., Liu, X., Yang, X.L. \& Suh, Y. (2014) A common variant in pre-miR-146 is associated with coronary artery disease risk and its mature miRNA expression. Mutat. Res. Fundam. Mol. Mech. Mutagen., 761, 15-20.

Xu, T., Zhu, Y., Wei, Q.K., Yuan, Y., Zhou, F., Ge, Y.Y., Yang, J.R., Su, H. \& Zhuang, S.M. (2008) A functional polymorphism in the miR-146a gene is associated with the risk for hepatocellular carcinoma. Carcinogenesis, 29, 2126-2131.

Yu, H., Gao, G., Jiang, L., Guo, L., Lin, M., Jiao, X., Jia, W. \& Huang, J. (2013) Decreased expression of miR-218 is associated with poor prognosis in patients with colorectal cancer. Int. J. Clin. Exp. Pathol., 6, 2904-2911.

Yu, H., Jiang, L., Sun, C., Guo, L., Lin, M., Huang, J. \& Zhu, L. (2014) Decreased circulating miR-375: a potential biomarker 
for patients with non-small-cell lung cancer. Gene, 534, 60-65.

Zhang, X., Chen, Q., He, C., Mao, W., Zhang, L., Xu, X., Zhu, J. \& Chen, B. (2012) Polymorphisms on $8 \mathrm{q} 24$ are associated with lung cancer risk and survival in Han Chinese. PLoS One, 7, e41930.

Zhi, H., Wang, L., Ma, G., Ye, X., Yu, X., Zhu, Y., Zhang, Y.,
Zhang, J. \& Wang, B. (2012) Polymorphisms of miRNAs genes are associated with the risk and prognosis of coronary artery disease. Clin. Res. Cardiol., 101, 289-296.

Zhou, B., Rao, L., Peng, Y., Wang, Y., Chen, Y., Song, Y. \& Zhang, L. (2010) Common genetic polymorphisms in pre-microRNAs were associated with increased risk of dilated cardiomyopathy. Clin. Chim. Acta, 411, 1287-1290. 